\title{
(c) OPEN ACCESS \\ Intracellular and non-neuronal targets of voltage-gated potassium channel complex antibodies
}

\author{
Bethan Lang, ${ }^{1}$ Mateusz Makuch, ${ }^{1}$ Teresa Moloney, ${ }^{1}$ Inga Dettmann, ${ }^{2}$ \\ Swantje Mindorf, ${ }^{2}$ Christian Probst, ${ }_{1}{ }^{2}$ Winfried Stoecker, ${ }^{2}$ Camilla Buckley, ${ }^{1}$ \\ Charles R Newton, ${ }^{3} \mathrm{M}$ Isabel Leite, ${ }^{1}$ Paul Maddison, ${ }_{1}^{4}$ Lars Komorowski, ${ }^{2}$ \\ Jane Adcock, ${ }^{1}$ Angela Vincent, ${ }^{1}$ Patrick Waters, ${ }^{1}$ Sarosh R Irani ${ }^{1}$
}

\begin{abstract}
- Additional material is published online only. To view please visit the journal online (http://dx.doi.org/10.1136/ jnnp-2016-314758)
\end{abstract}

'Nuffield Department of Clinical Neurosciences, University of Oxford, Oxford, UK

Institute for Experimental Immunology, Lubeck, Germany ${ }^{3}$ Department of Psychiatry, University of Oxford, Oxford, UK ${ }^{4}$ Department of Neurology, Queen's Medical Centre, Nottingham, UK

\section{Correspondence to} Professor Sarosh R Irani, West Wing, Level 6, John Radcliffe Hospital, Oxford OX3 9DU, UK; sarosh.irani@ndcn.ox.ac.uk

Received 25 August 2016 Revised 3 November 2016 Accepted 30 November 2016 Published Online First 23 January 2017

\section{CrossMark}

To cite: Lang $B$, Makuch $M$, Moloney T, et al. J Neurol Neurosurg Psychiatry 2017:88:353-361.

\section{ABSTRACT}

Objectives Autoantibodies against the extracellular domains of the voltage-gated potassium channel (VGKC) complex proteins, leucine-rich glioma-inactivated 1 (LGI1) and contactin-associated protein-2 (CASPR2), are found in patients with limbic encephalitis, faciobrachial dystonic seizures, Morvan's syndrome and neuromyotonia. However, in routine testing, VGKC complex antibodies without LGI1 or CASPR2 reactivities (double-negative) are more common than LGI1 or CASPR2 specificities.

Therefore, the target(s) and clinical associations of double-negative antibodies need to be determined.

Methods Sera ( $n=1131)$ from several clinically defined cohorts were tested for IgG radioimmunoprecipitation of radioiodinated $\alpha$-dendrotoxin $\left({ }^{125} \mid\right.$ - $\alpha$ DTX)-labelled VGKC complexes from mammalian brain extracts. Positive samples were systematically tested for live hippocampal neuron reactivity, Ig $\lg$ precipitation of ${ }^{125} \mathrm{I}-\alpha \mathrm{DTX}$ and ${ }^{125}$ - $\alpha$ DTX-labelled Kv1 subunits, and by cell-based assays which expressed Kv1 subunits, LGI1 and CASPR2.

Results VGKC complex antibodies were found in 162 of $1131(14 \%)$ sera. 90 of these (56\%) had antibodies targeting the extracellular domains of LGI1 or CASPR2. Of the remaining 72 double-negative sera, $10(14 \%)$ immunoprecipitated ${ }^{125}$ - $\alpha \mathrm{DTX}$ itself, and $27(38 \%)$ bound to solubilised co-expressed Kv1.1/1.2/1.6 subunits and/or Kv1.2 subunits alone, at levels proportionate to VGKC complex antibody levels $(r=0.57, p=0.0017)$. The sera with LGI1 and CASPR2 antibodies immunoprecipitated neither preparation. None of the $27 \mathrm{Kv1}$-precipitating samples bound live hippocampal neurons or Kv1 extracellular domains, but $16(59 \%)$ bound to permeabilised Kv1-expressing human embryonic kidney 293T cells. These intracellular Kv1 antibodies mainly associated with non-immune disease aetiologies, poor longitudinal clinical-serological correlations and a limited immunotherapy response.

Conclusions Double-negative VGKC complex antibodies are often directed against cytosolic epitopes of Kv1 subunits and occasionally against non-mammalian $\alpha \mathrm{DTX}$. These antibodies should no longer be classified as neuronal-surface antibodies. They consequently lack pathogenic potential and do not in themselves support the use of immunotherapies.

\section{INTRODUCTION}

Radioiodinated $\alpha$-dendrotoxin $\left({ }^{125} \mathrm{I}-\alpha \mathrm{DTX}\right)$ binds to neuronal voltage-gated potassium channels (VGKC) of the Shaker-family (Kv1.1, Kv1.2 and
Kv1.6), ${ }^{1}$ and was used to label these channels in the radioimmunoassay which first identified VGKC complex autoantibodies in patients with neuromyotonia (NMT), ${ }^{2}$ and then in Morvan's syndrome $(\mathrm{MoS}),{ }^{3}$ limbic encephalitis (LE) ${ }^{4} 5$ and faciobrachial dystonic seizures (FBDS). ${ }^{6} 7$ These autoantibodies were assumed to be directed against the Kv1 subunits themselves, but subsequent studies showed that the Kv1 subunits were part of a multiprotein neuronal complex that includes leucine-rich glioma inactivated 1 (LGI1), contactin-associated protein 2 (CASPR2) and contactin-2. Almost all of the antibodies from patients with LE, FBDS or MoS, and some with NMT, are directed against the extracellular domains of LGI1 or CASPR2, and the antibodies often co-immunoprecipitate the ${ }^{125}$ I- $\alpha$ DTX-labelled Kv1 subunits from brain extracts. $^{7-10}$ Patients with LGI1 or CASPR2 antibodies often respond very well to immunotherapies, and their antibody levels broadly correlate with clinical status. $^{711} 12$

Therefore, there has been an increasing interest in diagnosing these autoimmune neurological diseases, ${ }^{13}{ }^{14}$ leading to large numbers of requests for testing in patients who are unlikely to have welldefined autoimmune syndromes. This has generated an increase in the number of patient serum IgGs which precipitate the VGKC complex but lack LGI1 or CASPR2 reactivity ('double-negative' samples). These double-negatives can account for up to $80 \%$ of samples with positive VGKC complex antibodies in studies which most closely recapitulate clinical practice. ${ }^{15}{ }^{16}$ Moreover, the clinical syndromes in the double-negative patients are diverse and include patients with pain syndromes ${ }^{17}$ status epilepticus, ${ }^{18}$ acute and chronic epilepsies, ${ }^{19} 20$ inflammatory polyradiculopathies, ${ }^{21}$ children with a variety of neuroinflammatory diseases, ${ }^{22}$ systemic and central nervous system (CNS)-directed infections, ${ }^{23}$ a few patients with Creutzfeldt-Jakob disease, ${ }^{24}$ and up to $5 \%$ of elderly clinic controls. ${ }^{4}$ This clinical heterogeneity has questioned both the pathological relevance of the antibodies and the justification for immunotherapies in these patients. ${ }^{13} 141625$

Some studies have suggested that higher titres of the double-negative VGKC complex antibodies help to increase the likelihood of pathogenicity. ${ }^{22}{ }^{26}$ However, until now, the few available 
studies of double-negative patients have classified these patients by their clinical features and relied on arbitrary non-validated diagnostic criteria, and the subjective retrospective response to immunotherapies. ${ }^{16} \quad 25 \quad 26$ Here, to definitively determine whether double-negative VGKC complex antibodies have pathogenic potential, we explored the epitopes they bound, their titres and clinical associations across a large variety of clinical syndromes.

\section{METHODS}

Patients studied

To assess the frequencies of VGKC complex antibodies in a large number of varied patient phenotypes, and include syndromes reported to associate with double-negative samples, 1131 sera were tested from nine groups, including those with: (1) known LE, FBDS, MoS or NMT, LGI1 or CASPR2 antibodies and VGKC complex antibody levels $>400 \mathrm{pM}$ $(n=84) ;^{78}(2)$ a consecutive clinic cohort known to have VGKC complex antibodies without LGI1/CASPR2 reactivities $(n=27$; detailed in online supplementary table S1, which included patients with encephalopathies $(n=10)$, NMT $(n=2)$, stiff person syndrome $(n=2)$, psychiatric conditions $(n=6)$, isolated amnesia $(n=2)$, Parkinson's disease dementia $(n=1)$, Guillain-Barre syndrome $(n=1)$ and neuropathic pain $(n=3))$; (3) adult-onset epilepsies $(n=582)$; (4) infectious diseases $(n=107$ : herpes simplex virus encephalitis $(n=29)$, varicella zoster virus encephalitis (VZVE, $\mathrm{n}=20$ ), measles encephalitis $(\mathrm{n}=30)$ and malaria $(\mathrm{n}=28,12$ with cerebral involvement)); (5) dysautonomia $(\mathrm{n}=95)$; (6) Lambert-Eaton myasthenic syndrome $(n=45)$; (7) Hu-antibodies $(n=78)$; (8) healthy smokers $(n=38)$ and (9) healthy laboratory controls $(n=75)$. Approval for antibody studies was from the Oxfordshire Regional Ethical Committee A (07/Q1604/28).

\section{Laboratory techniques}

VGKC complex antibodies were detected by a radioimmunoassay which uses ${ }^{125}$ I- $\alpha$ DTX (Perkin Elmer, USA) to label VGKC complexes from $2 \%$ digitonin-solubilised rabbit whole brain membranes. ${ }^{2}{ }^{4}$ In order to closely mimic these conditions, but detect antibodies exclusively against the $\alpha$ DTX-sensitive Kv1 subunits, Kv1.1-tranfected, Kv1.2-tranfected and Kv1.6-transfected human embryonic kidney 293T (HEK) cells were used in place of brain tissue to prepare the extracts. In other respects, the radioimmunoassays were identical. To see if results were confounded by antibodies binding the ${ }^{125} \mathrm{I}-\alpha \mathrm{DTX}$ itself, the tissue/ cell extracts were replaced by solubilisation buffer. In each case, $5 \mu \mathrm{L}$ of patient serum was incubated with $50 \mu \mathrm{L}$ brain extract, HEK cell extract or buffer overnight and precipitated with $50 \mu \mathrm{L}$ antihuman immunoglobulin G (IgG; Binding Site). The cut-off for positivity based on the mean plus three SDs of results from 20 healthy control sera was $100 \mathrm{pM}$ for VGKC complex antibodies, $80 \mathrm{pM}$ for Kv1 subunit antibodies and $137 \mathrm{pM}$ for antibodies against ${ }^{125} \mathrm{I}$ - $\alpha$ DTX alone.

Culture and staining procedures for live hippocampal neurons, and for live cell-based assays (CBAs) to detect antibodies against LGI1, CASPR2, contactin-2, Kv1.1, Kv1.2 and Kv1.6 were performed as described previously. ${ }^{8}$ To validate CBA results, flow cytometry was performed with live Kv1-transfected HEK cells incubated with patient serum (1:20), and bound-IgG detected using a phycoerythrin-conjugated antihuman IgG secondary antibody. Samples were analysed on a LSRII flow cytometer with FlowJo V.10.0.8 software. Fixed CBAs were performed (at Euroimmun AG, Lübeck, Germany) with Kv1-transfected HEK cells, after fixation with $1.8 \%$ formalin followed by pure acetone, using a 1:10 dilution of patient serum from coded vials, with unblinding after study completion.

Commercial antibodies against the extracellular domain of Kv1.1 (Neuromab, 75/105), and intracellular domains of Kv1.1 (Chemicon, AB9782), Kv1.2 (Millipore, AB5924 and Neuromab 75/008) and Kv1.6 (Chemicon, AB5184 and Neuromab 75/012) were used for immunoprecipitation and CBA studies. Commercial antibodies against the extracellular domains of Kv1.2 or Kv1.6 were not available. Statistics were performed using GraphPad Prism V.6, and individual tests are stated below.

\section{RESULTS}

Antibodies against the VGKC complex

Overall, across the varied cohorts, 162 of 1131 (14\%) patients had VGKC complex antibodies, mainly from the groups with known positivity (figure 1A). Live CBAs showed that 90 of 162 $(56 \%)$ patients had LGI1 or CASPR2 antibodies (4 with coexistent contactin-2 antibodies). Eighty-four of these 90 (90\%) patients had LE, FBDS, MoS and NMT, and 80 of the 90 sera $(87 \%)$, those with higher titres, also had $\operatorname{IgG}$ antibodies that bound the surface of hippocampal neurons. Of the remaining 72 (44\%) double-negative VGKC complex antibody-positive samples, only one-from a patient with LE in the clinic cohort (see online supplementary table S1) - bound to live hippocampal neurons, suggesting a possible novel surface antigen. Among the cohorts (3-9) without known VGKC complex antibodies, the percentage of positives ranged between $0 \%$ and $4 \%$, with the exception of the infectious group (19\%) some of which had very high titres (figure $1 \mathrm{~A}$ ).

\section{Antibodies against ${ }^{125}$ I- $\alpha$ DTX}

One possibility was that the double-negative sera bound to ${ }^{125} \mathrm{I}-\alpha \mathrm{DTX}$ which is used to radiolabel the VGKC complex. Indeed, 10 of the $72(14 \%)$ double-negative samples immunoprecipitated very high levels of the ${ }^{125} \mathrm{I}-\alpha \mathrm{DTX}$ itself (figure 1B), correlating broadly with the corresponding VGKC complex antibody titres (figure 1C, $r=0.54 ; \mathrm{p}=0.02$, Spearman's rank correlation). All these 10 were found in patients with infectious diseases (malaria $(n=4)$, cerebral malaria $(n=4)$, VZVE $(n=1)$ and measles encephalitis $(n=1))$. Similarly, three sera from a snake handler, with vocational exposure to $\alpha$ DTX, immunoprecipitated ${ }^{125} \mathrm{I}-\alpha \mathrm{DTX}$ (figure $1 \mathrm{~B}$ ). The remaining 62 double-negative samples without ${ }^{125}$ I- $\alpha$ DTX reactivity were carried forward to the next experiments.

\section{Antibodies against the $\alpha$ DTX binding VGKC subunits detected in solution}

To test selectively for binding to the Kv1 subunits themselves, under conditions of the VGKC complex antibody assay, ${ }^{125} \mathrm{I}-\alpha \mathrm{DTX}$-labelled digitonin extracts of Kv1-transfected HEK cells were examined. Strikingly, none of the LGI1 or CASPR2 antibody-positive sera precipitated the Kv1 subunits (figure $2 \mathrm{~A}$ ). In contrast, 27 of $62(44 \%)$ double-negative samples bound in solution to ${ }^{125} \mathrm{I}-\alpha \mathrm{DTX}$-labelled $\mathrm{Kv} 1.1 / 1.2 / 1.6$ heteromers only $(n=6),{ }^{125}$ I- $\alpha$ DTX-labelled Kv1.2 homomers only $(n=6)$ or to both $(n=15$; figure $2 \mathrm{~A}$ and see online supplementary figure $\mathrm{S} 1 \mathrm{~A})$. Furthermore, their binding to Kv1s correlated well with their corresponding VGKC complex antibody levels (figure 2A, $\mathrm{r}=0.57, \mathrm{p}=0.0017$, Spearman's rank correlation). Ten of these 27 samples (37\%) had VGKC complex antibody levels over $400 \mathrm{pM}$. No increase in binding was observed with additional co-transfection of postsynaptic density protein 95 (PSD95, see 

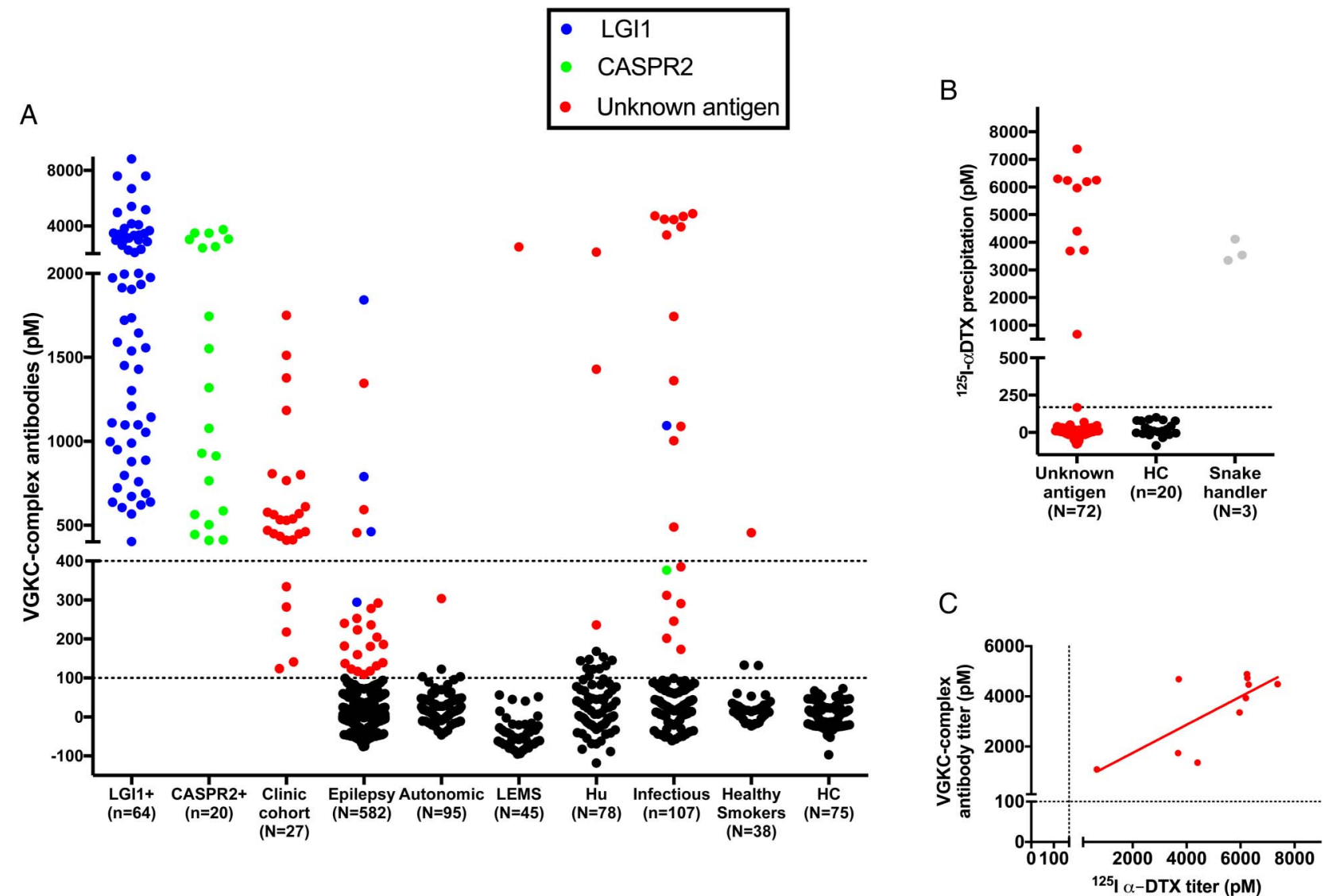

Figure 1 Detection of VGKC complex antibodies and antibodies to dendrotoxin. (A) VGKC complex antibodies were determined from 1131 samples, including those with known VGKC complex antibodies (both with $(n=84)$ and without $(n=27)$ LGI1 or CASPR2 reactivities), and unselected patients with adult-onset epilepsies, infectious diseases, autonomic syndromes, LEMS, Hu, healthy smokers and HC. Samples with LGI1 antibodies $(n=69)$, CASPR2 antibodies $(n=21)$ and all available samples with VGKC complex antibody levels above $100 \mathrm{pM}$ and unknown antigenic targets (red; $\mathrm{n}=72$ ) were carried forward to other assays. Dotted lines represent this cut-off and the $400 \mathrm{pM}$ cut-off from a previous study; ${ }^{4}$ (B) 10 of the 72 samples with unknown antigens immunoprecipitated substantial quantities of of ${ }^{125} \mathrm{I}-\alpha \mathrm{DTX}$ alone (dotted line at $137 \mathrm{pM}$ represents the mean plus three standard deviations from $20 \mathrm{HCs}$ ). Three serum samples from a snake handler (grey dots) also had antibodies to ${ }^{125}$ - $\alpha \mathrm{DTX} ;$; (C) ${ }^{125}$ - $\alpha \mathrm{\alpha TX}$ antibody levels correlated with their corresponding VGKC complex antibody levels ( $r=0.54, p=0.015$, Spearman's rank correlation). ${ }^{125}$ I- $\alpha$ DTX, radioiodinated $\alpha$-dendrotoxin; CASPR2, contactin-associated protein-2; HC, healthy controls; Hu, Hu antibodies; LEMS, Lambert-Eaton myasthenic syndrome; LGI1, leucine-rich glioma-inactivated 1; VGKC, voltage-gated potassium channel.

online supplementary figure S1B), a putative Kv1-clustering molecule. $^{27}$

Antibodies do not bind the extracellular domains of $\alpha \mathrm{DTX}$-sensitive VGKCs

The Kv1 reactivities available in solution could include intracellular or extracellular epitopes. To restrict detection to extracellular epitopes, live Kv1-transfected CBAs were tested. ${ }^{125} \mathrm{I}-\alpha \mathrm{DTX}$ surface-binding studies on live Kv1-transfected HEK cells (see online supplementary figure $\mathrm{S} 1 \mathrm{C}$ ) and commercial antibodies to the extracellular domain of Kv1.1 (figure 2B) confirmed adequate surface expression of Kv1.1, Kv1.2 and Kv1.6. However, none of the double-negative sera bound to live HEK cells transfected with individual Kv1.1, Kv1.2 or Kv1.6, or all three Kv1 subunits (representative example in figure 2B). To ensure maximal sensitivity, ${ }^{28}$ these negative results were confirmed using flow cytometry on the live HEK cells co-transfected with Kv1.1, Kv1.2 and Kv1.6: there was no evidence of surface binding in the 16 sera from figure $2 \mathrm{~A}$ with the highest Kv1 antibody radioimmunoassay values (see online supplementary figures S1D-F).
Antibodies bind the intracellular domains of $\alpha$ DTX-sensitive VGKCs

These results prove the existence of double-negative VGKC complex antibodies which bind Kv1 subunits in solution, but not Kv1 extracellular epitopes. Therefore, HEK cells expressing Kv1.1, Kv1.2 and Kv1.6 were fixed and permeabilised so that antibodies against intracellular epitopes could be detected. Commercial antibodies raised against intracellular sequences of all the $\alpha$ DTX-sensitive Kv1 subunits bound specifically to the appropriate fixed HEK cells (example in figure $2 \mathrm{C}$ and see online supplementary figure S2A,B), and their binding was abrogated after absorption of the commercial antibody with the immunising cytosolic Kv1 subunit peptide (shown for Kv1.2, see online supplementary figure S2A). This confirmed accessibility of antibodies to intracellular Kv1-subunit epitopes.

Subsequently, 175 coded sera were tested for binding to the fixed permeabilised Kv1-expressing cells. These included first samples of all double-negative patients without $\alpha$ DTX reactivity $(\mathrm{n}=62), 6$ with $\alpha \mathrm{DTX}$ antibodies, 57 sequential samples from the 27 patients with Kv1 antibodies demonstrated in solution (from figure 2A), patients with known LGI1 and CASPR2 antibodies $(n=20)$, and disease and healthy controls without VGKC 
A

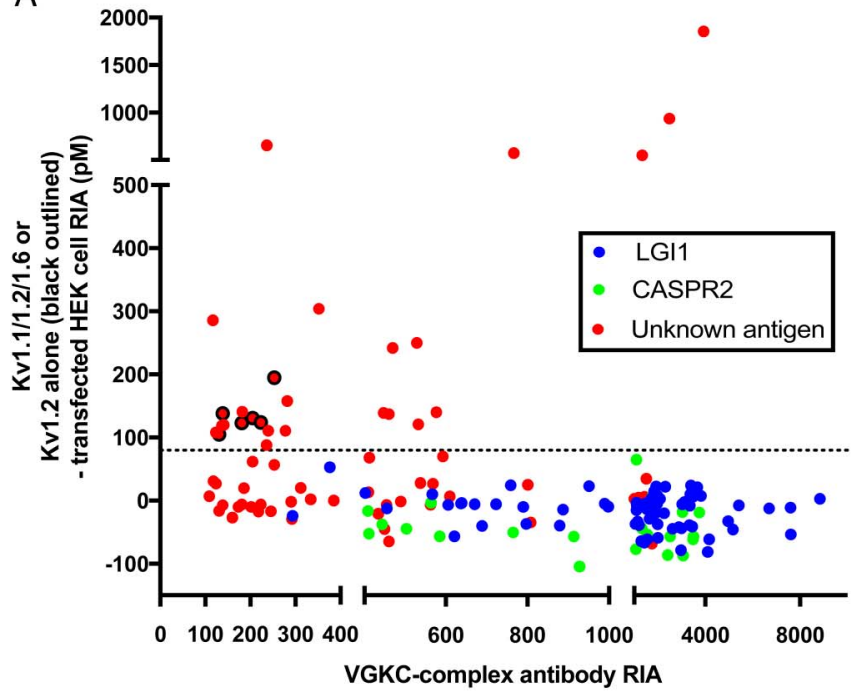

B

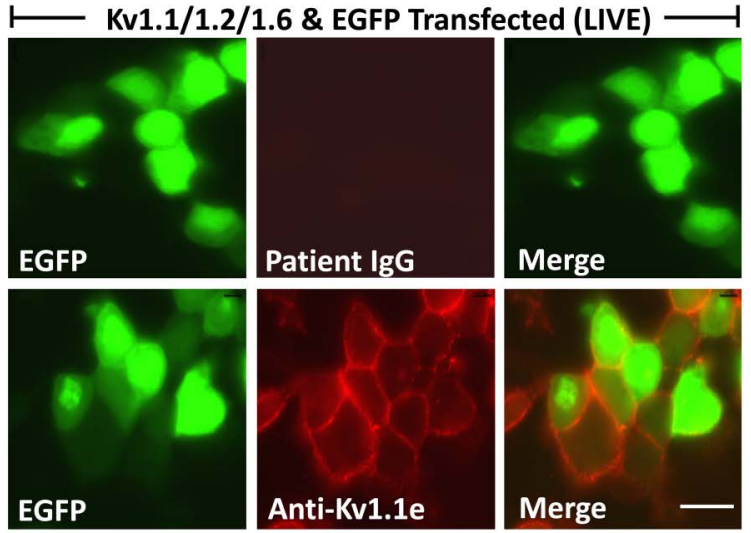

\section{C}

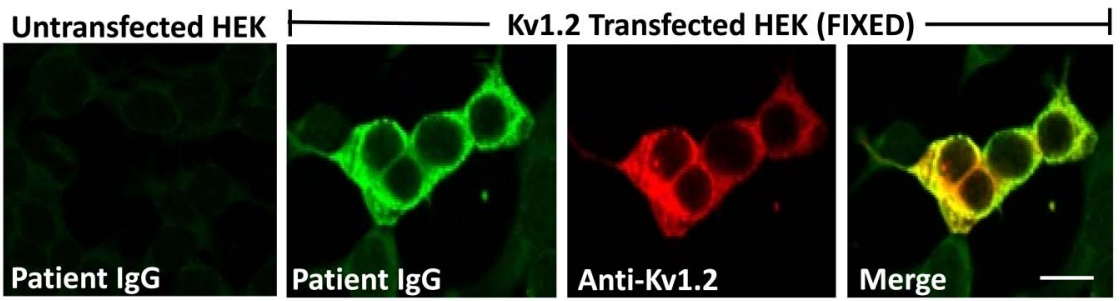

Figure 2 Kv1 antibodies target intracellular epitopes. (A) Twenty-seven of the remaining 62 patients with unknown VGKC complex antigenic targets precipitated either ${ }^{125} \mathrm{I}$ - $\alpha$ DTX-labelled Kv1.1/Kv1.2/Kv1.6 co-transfected HEK cell extracts (red circles) or ${ }^{125} \mathrm{I}$ - $\alpha$ DTX-labelled Kv1.2-transfected HEK cell extracts (red circles with black outline). No sera with LGI1 or CASPR2 antibodies showed positive results. HEK cells transfected with Kv1.1 alone or Kv1.6 alone did not bind ${ }^{125} \mathrm{I}-\alpha \mathrm{DTX}$ in solution; (B) a commercial antibody to the extracellular domain of Kv1.1 (anti-Kv1.1e) labelled the cell surface of live HEK cells co-transfected with Kv1.1, Kv1.2 and Kv1.6 (and enhanced green fluorescent protein (EGFP)). No patient antibodies ( $n=175$, including the 62 double-negative samples without $\alpha$ DTX reactivity) showed similar binding to these live cells or live cells expressing only one of these subunits; (C) binding to fixed Kv1-transfected HEK cells was seen using serum samples which precipitated Kv1s from solution. This co-localised with binding of commercial antibodies against the intracellular domain of Kv1.2 (anti-Kv1.2). Examples for Kv1.2 and Kv1.6 are shown in online supplementary figure S1C. Scale bar=10 microns. ${ }^{125} \mathrm{I}-\alpha \mathrm{DTX}$, radioiodinated $\alpha$-dendrotoxin; CASPR2, contactin-associated protein-2; HEK, human embryonic kidney 293T; LGI1, leucine-rich glioma-inactivated 1; VGKC, voltage-gated potassium channel.

complex antibodies $(n=30)$. Binding was observed in 41 of 175 $(23 \%)$ samples (examples in figure $2 \mathrm{C}$ and online supplementary figure S2A, B): all of these were from the group of 27 patients whose first sample immunoprecipitated Kv1.1/Kv1.2/ Kv1.6 subunits (from figure 2A, Mann-Whitney test, $\mathrm{p}<0.0001)$. The first available sample from 16 of these 27 patients bound to $\operatorname{Kv1.2~(n=9),~Kv1.6~(n=3),~Kv1.1~(n=1),~}$ $\mathrm{Kv1.1}, \mathrm{Kv1.2}$ and Kv1.6 $(\mathrm{n}=2)$, or both Kv1.2 and Kv1.6 $(n=1)$. These patient IgGs showed consistent co-localisation with the Kv1 commercial antibodies (see online supplementary figure S2B). Overall, of the 27 samples which precipitated ${ }^{125}$ I- $\alpha$ DTX-labelled Kv1 subunits in solution, those which did not show fixed CBA positivity tended towards lower VGKC complex levels (Mann-Whitney test, $\mathrm{p}=0.001$; see online supplementary figure S3A). The overall flow of samples and results by cohort is described in figure 3 , and the extracellular and intracellular molecular reactivities of the VGKC complex antibodies are summarised in figure 4A.

Correlations between Kv1 antibodies, clinical features and treatment responses

Serological and clinical details of the 27 patients with intracellular Kv1 antibodies are summarised in table 1 . There were 16 men and 11 women, with ages ranging from 18 to 85 years and no peak age at onset (figure 4B). Only 5 (19\%) had classical autoimmune syndromes (paraneoplastic, NMT or LE; figure 4C). The other patients had symptomatic or idiopathic generalised epilepsies $(n=5)$, neurodegenerative diseases $(n=2$, Parkinson's disease dementia and Alzheimer's disease), and 14 presented with conditions of unknown aetiology including cryptogenic epilepsies $(n=9)$, neuropathic pain $(n=2)$, chronic encephalopathy $(n=1)$, dysautonomia $(n=1)$ or spontaneously resolving amnesia $(n=1)$. In addition, one healthy smoker had intracellular Kv1 antibodies.

Despite this serological and clinical heterogeneity, 6 of the 9 (67\%) patients with cryptogenic epilepsies had Kv1.2-specific antibodies, both patients with neuropathic pain had Kv1.6 reactivities, and both patients with small cell lung carcinoma had antibodies directed against all three subunits (Kv1.1, Kv1.2 and Kv1.6).

Eleven of the 27 patients were administered immunotherapies and only $3(27 \%)$ showed a sustained clinical benefit. In addition, only 4 of the 19 (21\%) patients with serial serum samples demonstrated a relationship between intracellular antibody levels and clinical outcome (LE, NMT and 2 with cryptogenic epilepsies), while the remaining 15 demonstrated poor correlations. This contrasts with the majority of patients with LGI1 or CASPR2 antibodies (see online supplementary figure S3B-E). 


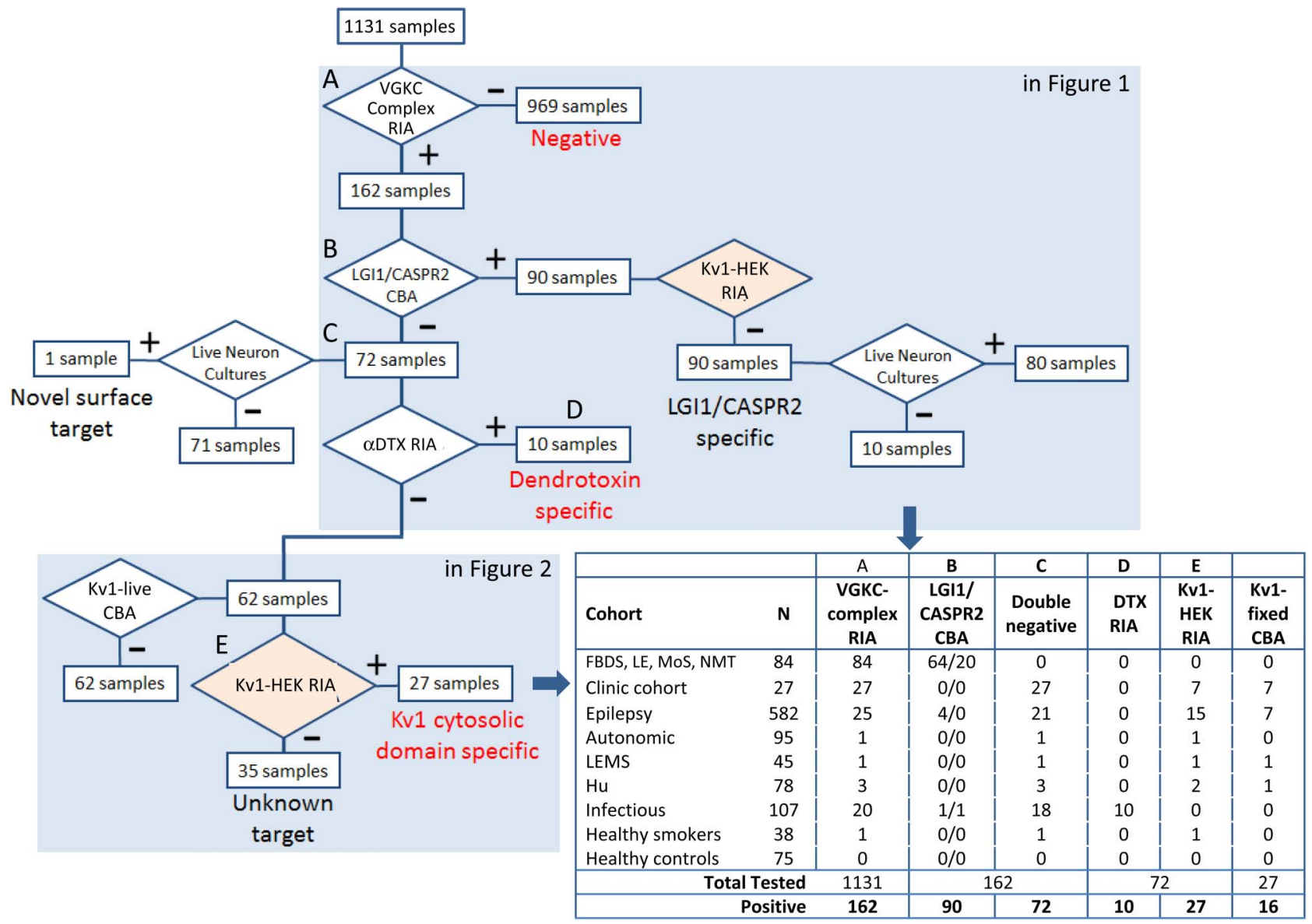

Figure 3 Summary of the sequential flow of assays through the study. As shown in figure 1A, 1131 samples were initially tested for VGKC complex antibodies by RIA (VGKC complex RIA, A) and subsequently using LGI1 and CASPR2 antibody live CBAs (B), live neuronal cultures (C) and precipitation of $\alpha$ DTX $(\alpha$ DTX RIA, D). As detailed in figure 2, double-negative samples were then tested for binding to the extracellular domains of live Kv1-tranfected HEK cells (Kv1-live CBA), for immunoprecipitations of ${ }^{125} \mathrm{I}-\alpha \mathrm{DTX}$-labelled Kv1-transfected HEK cells (Kv1-HEK RIA, E) and for binding to fixed permeabilised Kv1-transfected HEK cells (Kv1-fixed CBA). Cohorts are defined in more detail in the Methods section and online supplementary table S1. ${ }^{125}$ I- $\alpha$ DTX, radioiodinated $\alpha$-dendrotoxin; CASPR2, contactin-associated protein-2; CBA, cell-based assay; HEK, human embryonic kidney 293T; Hu, Hu antibodies; LEMS, Lambert-Eaton myasthenic syndrome; LGI1, leucine-rich glioma-inactivated 1; RIA, radioimmunoassay; VGKC, voltage-gated potassium channel.

Interestingly, in all individual patients, the targeted Kv1 subunit (s) and their levels relative to VGKC complex antibodies remained constant over time, strongly suggesting that these two assays were measuring the same antibody populations (see online supplementary figures S3 C-E).

\section{DISCUSSION}

Autoantibodies directed against the extracellular domains of LGI1 and CASPR2 usually associate with distinctive immunotherapy-responsive syndromes. Indeed, clinical and accumulating paraclinical data strongly suggest that they are directly pathogenic. $^{8} 911{ }^{29-31}$ In contrast, concerns have been raised about the clinical relevance of the double-negative VGKC complex antibodies that do not bind either of these proteins, particularly as they can be found in a proportion of patients with diseases which are unlikely to be of autoimmune aetiology. ${ }^{13} 14162225$ Studies which have interpreted the clinical relevance of such antibody results are limited by the inevitable difficulties in defining what constitutes an autoimmune disease. $^{16} 22 \quad 2532$ Ultimately, the definition of autoimmune neurological diseases relies on the demonstration of a pathogenic immune factor. Therefore, the findings described here are important because they use a systematic biochemical approach to demonstrate conclusively that a proportion of VGKC complex antibodies binds to intracellular VGKC epitopes, or to the non-mammalian-expressed $\alpha$ DTX. Discovery of these important antigenic targets should influence ongoing clinical practice, and prompt re-evaluation of several reports describing the clinical associations of patients with double-negative VGKC complex antibodies. ${ }^{15} 17-192633$ In this study, double-negative antibodies were observed in several non-autoimmune conditions including highly variable central and peripheral nervous system syndromes with limited responses to immunotherapies, and poor correlations were noted between clinical data and serial antibody levels. Taken together, these double-negative autoantibodies often have targets which are inaccessible or non-existent in vivo, and they appear to be associated with limited clinical relevance.

Overall, LGI1 and CASPR2 antibody CBAs conferred greater clinical utility and better sensitivity and specificity than VGKC complex antibody testing in providing a diagnosis and rationale for immunotherapy. This should prompt clinicians to use LGI1 and CASPR2 antibodies over VGKC complex antibodies as first-line testing for pathogenic antibody-associated immunotherapy-responsive syndromes, and limit immunotherapy 
A

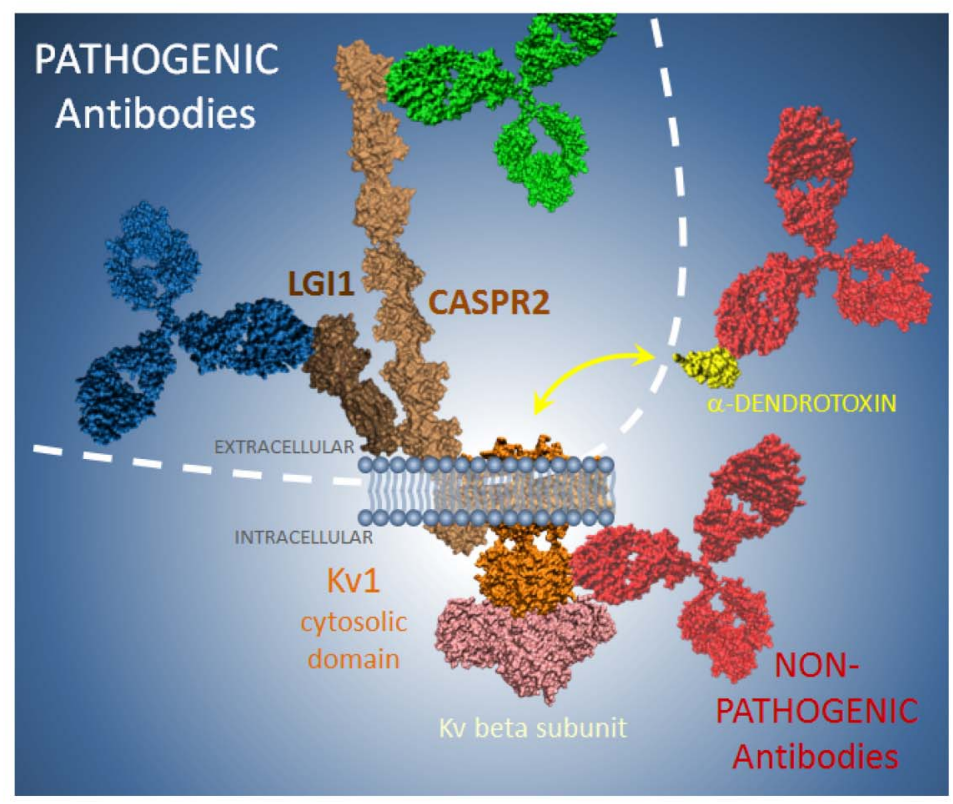

B

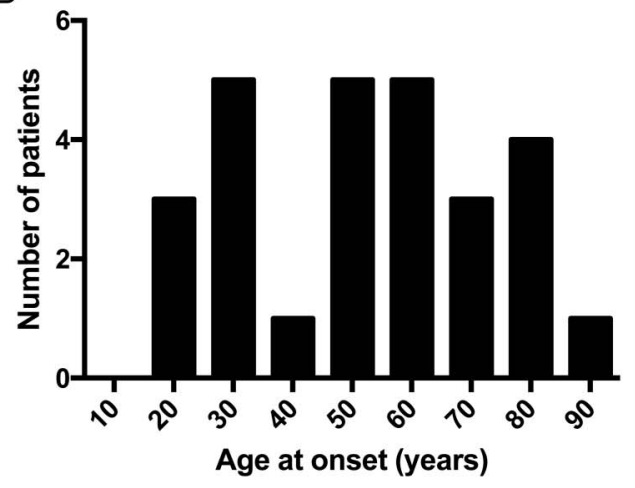

C

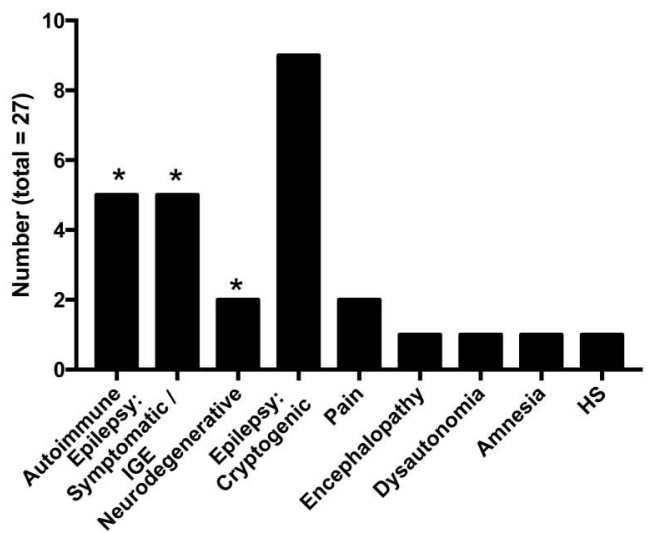

Figure 4 Molecular and clinical features associated with double-negative VGKC complex antibodies. (A) The illustration of study results demonstrates that the antibodies with pathogenic potential (blue and green) target the extracellular domains of LGI1 and CASPR2, respectively, whereas likely non-pathogenic antibodies (red) target the intracellular domain of Kv1 channels, especially Kv1.2, and the $\alpha$-dendrotoxin molecule itself (yellow), which is not present in mammalian tissue. Other intracellular targets may include the Kv- $\beta 2$ subunit (pink). (B) The patients with intracellular Kv1 antibodies had no clear peak age of onset, and (C) 12 showed varied, known diagnoses (*), and 15 had conditions of unknown aetiology, unlikely to be autoimmune. CASPR2, contactin-associated protein-2; HS, hippocampal sclerosis; LGI1, leucine-rich glioma-inactivated 1; VGKC, voltage-gated potassium channel.

administration to patients with double-negative antibodies and atypical clinical syndromes.

A striking finding was the discovery of antibodies against aDTX itself both in 10 patients with CNS infections and in a snake handler. $\alpha \mathrm{DTX}$ is a polypeptide toxin found in dendroaspis snake venoms and was essential for labelling the VGKC complex in the earliest studies. ${ }^{2} 451534$ Some of these studies included control tests to avoid detecting $\alpha$ DTX antibodies, ${ }^{15}$ but commercial VGKC complex antibody assays do not provide this specific control. The $\alpha$ DTX antibodies were almost exclusively in sera with high titres of VGKC complex antibodies. In contrast, the intracellular epitopes identified on the Kv1 subunits themselves, particularly on Kv1.2, were found both at high and low VGKC complex antibody levels. Therefore, this study shows that the antigenic target-rather than the VGKC complex antibody level-aids prediction of potential pathogenicity. This is consistent with the observation that while VGKC complex antibody levels associated with LGI1 and CASPR2 reactivities are often high, they can be low or even undetectable. ${ }^{8} 15253536$

Furthermore, this is the first clear demonstration of double-negative VGKC complex antibodies which target intracellular epitopes. This positive demonstration is important as we and others have previously hypothesised their existence, mainly inferring from the absence of live neuronal binding. ${ }^{13} 142225$
However, since a few disease-relevant LGI1 and CASPR2 antibody-positive samples had no detectable live hippocampal neuron binding, the absence of live neuronal binding should not necessarily imply the presence of intracellular reactivities. Indeed, it is most likely that not all surface neuronal proteins are expressed in a hippocampal cell culture system.

It is curious that the double-negative VGKC complex epitopes appear especially immunogenic: they are found after both human exposure to porcine brain aerosols and murine nasal immunisations with brain extracts, ${ }^{21}$ suggesting that these antibodies are easily induced. Collectively, alongside their presence in a variety of epilepsies, neurodegenerative diseases and idiopathic syndromes, these observations imply that double-negative antibodies arise secondary to diverse pathologies, where the primary pathological insult may not be immune. Indeed, perhaps non-immune causes of neuronal destruction are sufficient to release immunogenic Kv1 antigens and provoke autoantibody production. ${ }^{16} 22{ }^{26}$ Interestingly, the immunotherapy response observed in $27 \%$ of patients is very similar to the placebo response rates observed in several other neurological diseases, ${ }^{37}$ and much lower than those reported in LGI1 or CASPR2 antibody-associated syndromes. ${ }^{7-9} 11152935$ However, conclusions regarding treatment outcomes are only definitive after blinded interventional studies. Also, the demographics of the patients with Kv1 antibodies did not show any age or sex 
Table 1 Clinical-serological details of patients with antibodies against the intracellular aspects of Kv1.1, Kv1.2 and/or Kv1.6

\begin{tabular}{|c|c|c|c|c|c|c|c|}
\hline \multirow[b]{2}{*}{$\begin{array}{l}\text { Agel } \\
\text { Sex }\end{array}$} & \multicolumn{3}{|c|}{ Serological results } & \multirow[b]{2}{*}{ Clinical features } & \multirow[b]{2}{*}{$\begin{array}{l}\text { Disease } \\
\text { aetiology }\end{array}$} & \multirow[b]{2}{*}{ Outcome and treatments } & \multirow[b]{2}{*}{ Summary of clinical-serological correlations } \\
\hline & $\begin{array}{l}\text { VGKC complex } \\
\text { antibody (pM) }\end{array}$ & $\begin{array}{l}\text { Kv1-HEK } \\
\text { RIA }\end{array}$ & $\begin{array}{l}\text { Kv1-fixed } \\
\text { CBA }\end{array}$ & & & & \\
\hline 59/M & 1346 & + & 1.2 only & $\begin{array}{l}\text { Cryptogenic TLE with amnesia/ } \\
\text { depression }\end{array}$ & Unknown & $\begin{array}{l}\text { No sustained response to three AEDs and } \\
\text { CS }\end{array}$ & $\begin{array}{l}\text { Poor. Persistent VGKC-c Abs despite markedly varied SZ frequencies. } \\
\text { Online supplementary figure S3C }\end{array}$ \\
\hline $49 / \mathrm{F}$ & 767 & + & 1.2 only & NMT with EMG confirmation & Autoimmune & Limited response to AEDs and CS & Poor. Highest VGKC-c Abs during clinical remission \\
\hline $55 / \mathrm{M}$ & 533 & + & 1.2 only & NMT with EMG confirmation & Autoimmune & $\begin{array}{l}\text { Symptoms over } 8 \text { years despite AEDs/AZA/ } \\
\text { CS }\end{array}$ & Good. Patient symptomatic with persistent VGKC-c Abs \\
\hline 77/M & 470 & + & 1.2 only & $\begin{array}{l}\text { Chronic myelopathy/ } \\
\text { encephalopathy }\end{array}$ & Unknown & Transient response to CS and PLEX & Poor. High VGKC-c Abs despite symptom fluctuations over 3 years \\
\hline 21/M & 461 & + & 1.2 only & $\begin{array}{l}\text { Cryptogenic probable frontal lobe } \\
\text { epilepsy }\end{array}$ & Unknown & Ongoing SZs despite two AEDs & Poor. Online supplementary figure S3E \\
\hline $50 / M$ & 448 & + & 1.2 only & Isolated amnesia & Unknown & Spontaneous resolution over a few days & Poor. Abs reduced over 12 months. Symptoms resolved at 7 days \\
\hline 28/F & 278 & + & 1.2 only & Cryptogenic TLE & Unknown & SZ freedom after first AED & Poor. Persistent VGKC-c Abs during SZ freedom \\
\hline $62 / \mathrm{M}$ & 236 & + & 1.2 only & Cryptogenic TLE with amnesia & Unknown & SZs and amnesia at 5 years with 3 AEDs & Poor. VGKC-c Abs disappeared while SZs were ongoing \\
\hline $18 / \mathrm{M}$ & 141 & + & 1.2 only & Limbic encephalitis & Autoimmune & Good response to CS and PLEX & Good. VGKC-c Abs reduced and clinical improvement at 12 months \\
\hline $25 / \mathrm{F}$ & 529 & + & 1.6 only & $\begin{array}{l}\text { Diffuse neuropathic pain and } \\
\text { depression }\end{array}$ & Unknown & No response to opioids, CS or IVIG & Poor. High VGKC-c Abs despite marked fluctuations in symptoms \\
\hline 68/M & 240 & + & 1.6 only & Alzheimer's disease; one SZ & Degenerative & Ongoing fall in memory despite CS/IVIG & $\begin{array}{l}\text { Poor. Slight fall in VGKC-c Abs but marked reduction in memory over } \\
4 \text { years }\end{array}$ \\
\hline $34 / \mathrm{M}$ & 577 & + & 1.2 and 1.6 & Widespread neuropathic pain & Unknown & No response to CS, IVIG and PLEX & $\begin{array}{l}\text { Poor. Constant pain despite highly varied VGKC-c Abs. Online } \\
\text { supplementary figure S3D }\end{array}$ \\
\hline $71 / \mathrm{F}$ & 2489 & + & $\begin{array}{l}1.1,1.2 \text { and } \\
1.6\end{array}$ & NMT plus SCLC & Paraneoplastic & Palliative care only & NA. \\
\hline $67 / \mathrm{F}$ & 2120 & + & $\begin{array}{l}1.1,1.2 \text { and } \\
1.6\end{array}$ & $\begin{array}{l}\text { LEMS plus Hu antibody } \\
\text { neuropathy and SCLC }\end{array}$ & Paraneoplastic & Good response to CS & $\begin{array}{l}\text { Poor. Persistently high VGKC-c Abs over } 5 \text { years despite clinical } \\
\text { improvements }\end{array}$ \\
\hline $84 / \mathrm{F}$ & 282 & + & 1.1 only & Parkinson's disease dementia & Degenerative & No response to CS & Poor. VGKC-c Abs reduced over 12 months despite clinical worsening \\
\hline $58 / \mathrm{M}$ & 304 & + & Negative & Dysautonomia & Unknown & NA & NA \\
\hline $37 / \mathrm{M}$ & 253 & + & Negative & TLE related to left HS & Structural & NA & NA \\
\hline 59/M & 236 & + & Negative & Healthy smoker & Healthy & Not relevant & Not relevant \\
\hline $48 / \mathrm{F}$ & 223 & + & Negative & TLE related to left HS & Structural & NA & NA \\
\hline $85 / F$ & 205 & + & Negative & Cryptogenic focal motor SZs & Unknown & $\begin{array}{l}\text { SZ free on } 1 \text { AED. Amnesia and anxiety } \\
\text { benefited from CS/IVIG }\end{array}$ & $\begin{array}{l}\text { Poor. VGKC-c Abs disappeared over } 1 \text { year; SZ freedom at } 2 \text { years. } \\
\text { VGKC-c Abs returned at } 4 \text { years without SZs }\end{array}$ \\
\hline $33 / \mathrm{F}$ & 182 & + & Negative & Cryptogenic TLE & Unknown & SZ freedom after second AED & Moderate. VGKC-c Abs reduced over 6 months and SZ freedom at 1 year \\
\hline $76 / \mathrm{M}$ & 181 & + & Negative & Cryptogenic epilepsy & Unknown & SZ freedom with 1 AED & Poor. SZ freedom at 6 months; VGKC-c Abs sampled after 15 years \\
\hline $54 / \mathrm{F}$ & 139 & + & Negative & $\begin{array}{l}\text { Epilepsy after childhood } \\
\text { meningitis }\end{array}$ & Structural & NA & NA \\
\hline 77/M & 123 & + & Negative & TLE secondary to CVA & Structural & Ongoing SZs at 4 years & NA \\
\hline
\end{tabular}


bias, quite different from the occurrence of LGI1 antibody LE in later years or the male predominance of CASPR2 antibodies. $^{8} 91129$ Alongside the 5\% rate of double-negative VGKC complex antibodies in healthy individuals, ${ }^{4}$ these observations suggest that double-negative reactivities have very limited clinical significance.

On the other hand, we noted relationships between specific Kv1 subunit reactivities and clinical syndromes, particularly Kv1.2 antibodies in cryptogenic epilepsies, Kv1.6 antibodies in the two patients with neuropathic pain, and Kv1.1, Kv1.2 and Kv1.6 antibodies in paraneoplastic syndromes. These preliminary findings suggest that specific patterns of Kv1 reactivities may be generated in response to certain pathologies. Indeed, a few patients with classical VGKC complex antibody-associated syndromes, such as NMT and LE, did show good antibody-clinical correlations, and in these patients the Kv1 antibodies may be markers of a coexistent pathogenic antibody. ${ }^{38} 39$ However, while many of the clinic cohort had an underlying immune basis to their neurological syndrome, ascertainment of patients attending a specialist autoimmune clinic may well carry this intrinsic bias.

In summary, our results show that many double-negative VGKC complex antibodies lack pathogenic potential, and that direct examination of LGI1 and CASPR2 antibodies is more informative than first-line VGKC complex antibody

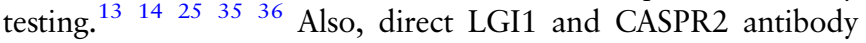
CBAs appear more sensitive than VGKC complex antibody or live neuronal antibody determination. ${ }^{25} 3536$ Therefore, direct LGI1 and CASPR2 antibody assays are the preferred tests for clinical purposes. This approach should limit the use of unnecessary immunotherapies in double-negative patients.

Until all centres update their practices, treatment of double-negative cases should be limited to those with a high pretest probability of an underlying autoimmune condition. However, for research purposes, it may be useful for further studies to identify the remaining, likely intracellular, double-negative VGKC complex antigens such as the $\mathrm{Kv}-\beta 2$ subunit and PSD95, and explore whether they are useful biomarkers of traditionally non-immune or immune neurological syndromes.

Funding BL is supported by Epilepsy Research UK (ERUK; P1201); SRI is supported by a Wellcome Trust Intermediate Fellowship (104079/Z/14/Z), BMA Research Grants-Vera Down grant (2013), the Fulbright UK-US commission and the MS society. Research in the Neuroimmunology Laboratory is supported by the Oxford NIHR Biomedical Research Centre. PW and MIL are supported by the NHS National Specialized Commissioning Group for Neuromyelitis optica, UK.

Competing interests $\mathrm{AV}, \mathrm{SRI}, \mathrm{BL}$ and $\mathrm{PW}$ are coapplicants and receive royalties on patent application WO/2010/046716 entitled 'Neurological Autoimmune Disorders'. The patent has been licensed to Euroimmun AG for the development of assays for LGI1 and other VGKC-complex antibodies. BL and SRI had full access to all of the data in the study and take responsibility for the integrity of the data and the accuracy of the data analysis.

Ethics approval Oxfordshire Regional Ethical Committee A (RECA)

Provenance and peer review Not commissioned; externally peer reviewed.

Open Access This is an Open Access article distributed in accordance with the terms of the Creative Commons Attribution (CC BY 4.0) license, which permits others to distribute, remix, adapt and build upon this work, for commercial use, provided the original work is properly cited. See: http://creativecommons.org/licenses/ by/4.0/

\section{REFERENCES}

1 Shamotienko OG, Parcej DN, Dolly JO. Subunit combinations defined for K+ channel Kv1 subtypes in synaptic membranes from bovine brain. Biochemistry 1997;36:8195-201.
2 Shillito $P$, Molenaar PC, Vincent A, et al. Acquired neuromyotonia: evidence for autoantibodies directed against K+ channels of peripheral nerves. Ann Neurol 1995;38:714-22.

3 Liguori R, Vincent A, Clover L, et al. Morvan's syndrome: peripheral and central nervous system and cardiac involvement with antibodies to voltage-gated potassium channels. Brain 2001:124:2417-26.

4 Vincent A, Buckley C, Schott JM, et al. Potassium channel antibody-associated encephalopathy: a potentially immunotherapy-responsive form of limbic encephalitis. Brain 2004;127:701-12.

5 Thieben MJ, Lennon VA, Boeve BF, et al. Potentially reversible autoimmune limbic encephalitis with neuronal potassium channel antibody. Neurology 2004:62:1177-82.

6 Irani SR, Buckley C, Vincent A, et al. Immunotherapy-responsive seizure-like episodes with potassium channel antibodies. Neurology 2008;71:1647-8.

7 Irani SR, Michell AW, Lang B, et al. Faciobrachial dystonic seizures precede Lgi1 antibody limbic encephalitis. Ann Neurol 2011;69:892-900.

8 Irani SR, Alexander S, Waters $\mathrm{P}$, et al. Antibodies to Kv1 potassium channel-complex proteins leucine-rich, glioma inactivated 1 protein and contactin-associated protein-2 in limbic encephalitis, Morvan's syndrome and acquired neuromyotonia. Brain 2010;133:2734-48.

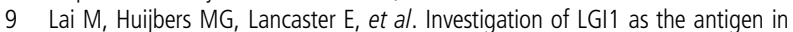
limbic encephalitis previously attributed to potassium channels: a case series. Lancet Neurol 2010;9:776-85.

10 Irani SR, Pettingill P, Kleopa KA, et al. Morvan syndrome: clinical and serological observations in 29 cases. Ann Neurol 2012;72:241-55.

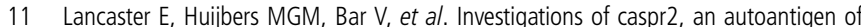
encephalitis and neuromyotonia. Ann Neurol 2011;69:303-11.

12 Shin YW, Lee ST, Shin JW, et al. VGKC-complex/LGI1-antibody encephalitis: clinical manifestations and response to immunotherapy. J Neuroimmunol 2013;265:75-81.

13 Irani SR, Gelfand JM, Al-Diwani A, et al. Cell-surface central nervous system autoantibodies: clinical relevance and emerging paradigms. Ann Neurol 2014;76:168-84

14 Leypoldt F, Armangue T, Dalmau J. Autoimmune encephalopathies. Ann N Y Acad Sci 2015;1338:94-114.

15 Klein CJ, Lennon VA, Aston PA, et al. Insights from LGI1 and CASPR2 potassium channel complex autoantibody subtyping. JAMA Neurol 2013;70:229-6.

16 Paterson RW, Zandi MS, Armstrong R, et al. Clinical relevance of positive voltage-gated potassium channel (VGKC)-complex antibodies: experience from a tertiary referral centre. J Neurol Neurosurg Psychiatry 2014;85:625-30.

17 Klein CJ, Lennon VA, Aston PA, et al. Chronic pain as a manifestation of potassium channel-complex autoimmunity. Neurology 2012;79:1136-44.

18 Suleiman J, Brenner T, Gill D, et al. VGKC antibodies in pediatric encephalitis presenting with status epilepticus. Neurology 2011;76:1252-5.

19 Brenner T, Sills GJ, Hart Y, et al. Prevalence of neurologic autoantibodies in cohorts of patients with new and established epilepsy. Epilepsia 2013;54:1028-35.

20 Toledano M, Britton JW, McKeon A, et al. Utility of an immunotherapy trial in evaluating patients with presumed autoimmune epilepsy. Neurology 2014:82:1578-86.

21 Meeusen JW, Klein CJ, Pirko I, et al. Potassium channel complex autoimmunity induced by inhaled brain tissue aerosol. Ann Neurol 2012;71:417-26.

22 Hacohen $Y$, Singh R, Rossi $M$, et al. Clinical relevance of voltage-gated potassium channel-complex antibodies in children. Neurology 2015;85:967-75.

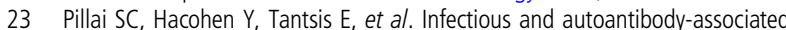
encephalitis: clinical features and long-term outcome. Pediatrics 2015;135 e974-84.

24 Rossi M, Mead S, Collinge J, et al. Neuronal antibodies in patients with suspected or confirmed sporadic Creutzfeldt-Jakob disease: table 1. J Neurol Neurosurg Psychiatry 2015;86:692-4

25 van Sonderen A, Schreurs MWJ, de Bruijn MAAM, et al. The relevance of VGKC positivity in the absence of LGI1 and Caspr2 antibodies. Neurology 2016:86:1692-9.

26 Olberg $H$, Haugen $M$, Storstein A, et al. Neurological manifestations related to level of voltage-gated potassium channel antibodies. J Neurol Neurosurg Psychiatry 2013:84:941-3.

27 Horresh I, Poliak S, Grant S, et al. Multiple molecular interactions determine the clustering of Caspr2 and Kv1 channels in myelinated axons. J Neurosci 2008;28:14213-22.

28 Waters PJ, McKeon A, Leite MI, et al. Serologic diagnosis of NMO: a multicenter comparison of aquaporin-4-IgG assays. Neurology 2012;78:665-71-discussion669.

29 Flanagan EP, Kotsenas AL, Britton JW, et al. Basal ganglia T1 hyperintensity in LGI1-autoantibody faciobrachial dystonic seizures. Neurol Neuroimmunol Neuroinflamm 2015:2:e161-1.

30 Ohkawa T, Fukata Y, Yamasaki M, et al. Autoantibodies to epilepsy-related LGI1 in limbic encephalitis neutralize LGI1-ADAM22 interaction and reduce synaptic AMPA receptors. J Neurosci 2013:33:18161-74.

31 Lalic T, Pettingill $P$, Vincent $A$, et al. Human limbic encephalitis serum enhances hippocampal mossy fiber-CA3 pyramidal cell synaptic transmission. Epilepsia 2011;52:121-31. 
32 Graus F, Titulaer MJ, Balu R, et al. A clinical approach to diagnosis of autoimmune encephalitis. Lancet Neurol 2016;15:391-404.

33 McKnight $K$, Jiang $Y$, Hart $Y$, et al. Serum antibodies in epilepsy and seizure-associated disorders. Neurology 2005:65:1730-6.

34 Hart IK, Waters C, Vincent A, et al. Autoantibodies detected to expressed K+ channels are implicated in neuromyotonia. Ann Neurol 1997;41:238-46.

35 Irani SR, Stagg CJ, Schott JM, et al. Faciobrachial dystonic seizures: the influence of immunotherapy on seizure control and prevention of cognitive impairment in a broadening phenotype. Brain 2013;136:3151-62.
36 Becker EBE, Zuliani L, Pettingill R, et al. Contactin-associated protein-2 antibodies in non-paraneoplastic cerebellar ataxia. J Neurol Neurosurg Psychiatry 2012;83:437-40.

37 Bittar C, Nascimento OJM. Placebo and nocebo effects in the neurological practice. Arq Neuro Psiquiatr 2015;73:58-63.

38 Boronat A, Sabater L, Saiz A, et al. GABA(B) receptor antibodies in limbic encephalitis and anti-GAD-associated neurologic disorders. Neurology 2011;76:795-800.

39 Pettingill P, Kramer HB, Coebergh JA, et al. Antibodies to GABAA receptor $\alpha 1$ and $\gamma 2$ subunits: clinical and serologic characterization. Neurology 2015;84:1233-41. 